\title{
A Ka-Band CMOS Wilkinson Power Divider Using Synthetic Quasi-TEM Transmission Lines
}

\author{
Meng-Ju Chiang, Student Member, IEEE, Hsien-Shun Wu, Member, IEEE, and \\ Ching-Kuang C. Tzuang, Fellow, IEEE
}

\begin{abstract}
This work presents a Ka-band two-way 3 dB Wilkinson power divider using synthetic quasi-transverse electromagnetic (TEM) transmission lines (TLs). The synthetic quasi-TEM TL, also called complementary-conducting-strip TL (CCS TL), is theoretically analyzed. The equivalent TL model, whose production is based on the extracted results, is applied to the power divider design. The prototype is fabricated by the standard $0.18 \mu \mathrm{m}$ 1P6M CMOS technology, showing the circuit size of $210.0 \mu \mathrm{m} \times 390.0 \mu \mathrm{m}$ without contact pads. The measurement results, which match the $50 \Omega$ system, reveal perfect agreements with those of the simulations. The comparison reveals the following characteristics. The divider exhibits an equal power-split with the insertion losses $\left(S_{21}\right.$ and $\left.S_{31}\right)$ of $3.65 \mathrm{~dB}$. The return losses $\left(S_{11}\right.$, $S_{22}$ and $S_{33}$ ) of the prototype are higher than $10.0 \mathrm{~dB}$ from 30.0 to $40.0 \mathrm{GHz}$.
\end{abstract}

Index Terms-Millimeter-wave circuit, radio frequency (RF) CMOS, synthetic transmission line (TL), Wilkinson power divider.

\section{INTRODUCTION}

$\mathbf{T}$ HE characteristics of the power divider, such as power division and divider bandwidth, can be exactly synthesized based on the equivalent transmission line (TL) network [1], [2]. A literature survey shows such TL-based components can be fully realized using advanced monolithic technologies. The intrinsic loss of the power divider can be one $\mathrm{dB}$ lower by using the silicon-based micromachining [3], [4], high resistivity substrate (HRS) [5], [6] and multilayer GaAs fabrications [7]-[9]. On the other hand, the size of the monolithic power divider can be smaller than $5.5 \times 10^{-4} \lambda_{0}^{2}$ using 3-D monolithic microwave integrated circuit (MMIC) technology at $20.0 \mathrm{GHz}$ [9]. Moreover, this work uses the standard CMOS technology to design a Ka-band two-way power divider for achieving low-loss and circuit miniaturization simultaneously. By applying the guiding characteristics of the proposed synthetic quasi-TEM TL presented in Section II, the prototype is designed and fabricated by the standard $0.18 \mu \mathrm{m}$ 1P6M CMOS technology. Section III reports both theoretical and measured results. The intrinsic losses $\left(S_{21}\right.$ and $\left.S_{31}\right)$ of the divider are $0.65 \mathrm{~dB}$, and the return losses $\left(S_{11}, S_{22}\right.$ and $\left.S_{33}\right)$ of the prototype are higher than $10.0 \mathrm{~dB}$ from 30.0 to $40.0 \mathrm{GHz}$. The chip area, as shown in Fig. 1, is $210.0 \mu \mathrm{m}$ $\times 390.0 \mu \mathrm{m}\left(1.1 \times 10^{-3} \lambda_{0}^{2}\right)$. Finally, Section IV concludes the letter.

Manuscript received May 22, 2007; revised August 14, 2007. The work was supported by the National Science Council of Taiwan, R.O.C., under Grants NSC 95-2752-E-002-009-PWE and NSC 95-2221-E-002-084-MY2.

The authors are with the Graduate Institute of Communication Engineering, National Taiwan University, Taipei 106, Taiwan, R.O.C (e-mail: d93942008@ntu.edu.tw; cktzuang@cc.ee.ntu.edu.tw).

Digital Object Identifier 10.1109/LMWC.2007.910475

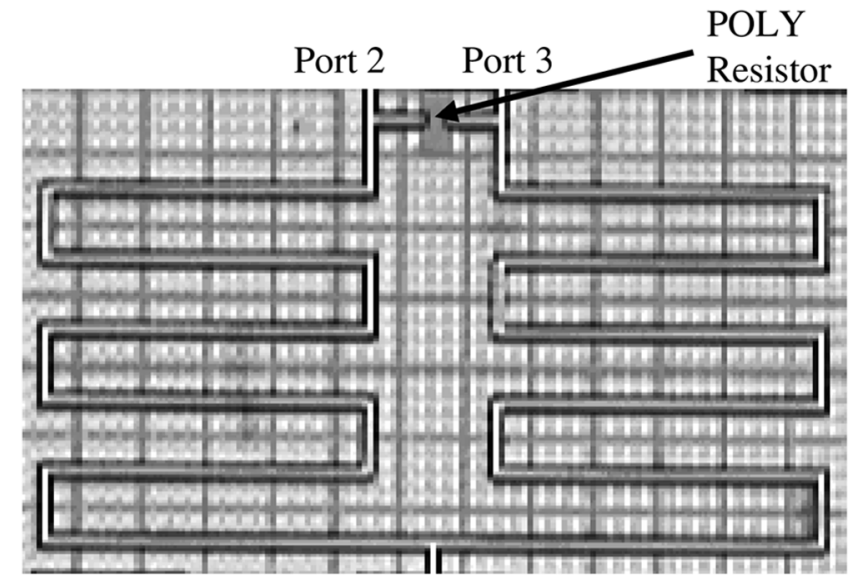

Port 1

Fig. 1. Prototype of CMOS Ka-band CCS-based power divider. Chip size without contact pads is $210.0 \mu \mathrm{m} \times 390.0 \mu \mathrm{m}$. The line width of the CCS TL is $4.0 \mu \mathrm{m}$. The total length of two CCS TLs is $2160.0 \mu \mathrm{m}$. The details of unit cell are shown in Fig. 2.

\section{GUIDING PROPERTIES OF THE COMPLEMENTARY-CONDUCTING-STRIP TRANSMISSION LINE (CCS TL)}

The concept of complementary-conducting-strip TL (CCS TL) was recently reported and successfully employed to miniaturize radio frequency integrated circuits (RFICs) [10], [11]. The CCS TL is produced by connecting unit cells and meanders in a two-dimensional plane. The design parameters of the unit cell include the periodicity of the unit cell, the area of the mesh ground plane, central patch, and the width of the connecting arms, etc., [10], [11]. Fig. 2 shows a typical example of CMOS CCS TL with a characteristic impedance of $71.8 \Omega$. The signal trace of the CCS TL is realized by the top metal and the mesh ground plane is constructed by stacking $M_{1}$ and $M_{2}$ metal layers. Two of design parameters, including of the line width of the signal trace and the ratio of $P$ and $W_{h}$, are applied to synthesize the characteristic impedance of the CCS TL in Fig. 2.

The theoretical analysis by using full-wave EM simulator Ansoft HFSS investigates the guiding characteristics of the CCS TL. The following structural parameters of the unit cells are applied to the electromagnetic (EM) simulations. The periodicity $(P)$ of the unit cell of the CCS TL is $30.0 \mu \mathrm{m}$ and the mesh area $\left(W_{h}\right)$ is $28.0 \mu \mathrm{m}$. The line-width $(S)$ of the signal path is $4.0 \mu \mathrm{m}$, the thickness of the signal path is $2.0 \mu \mathrm{m}$ and the effective thickness of the mesh ground plane is $2.0 \mu \mathrm{m}$. The dielectric constant of the inter-media-dielectric (IMD) is 4.0 with a thickness of $5.25 \mu \mathrm{m}$ and the relative dielectric constant of the silicon substrate is 11.9 . The thickness and conductivity of the 


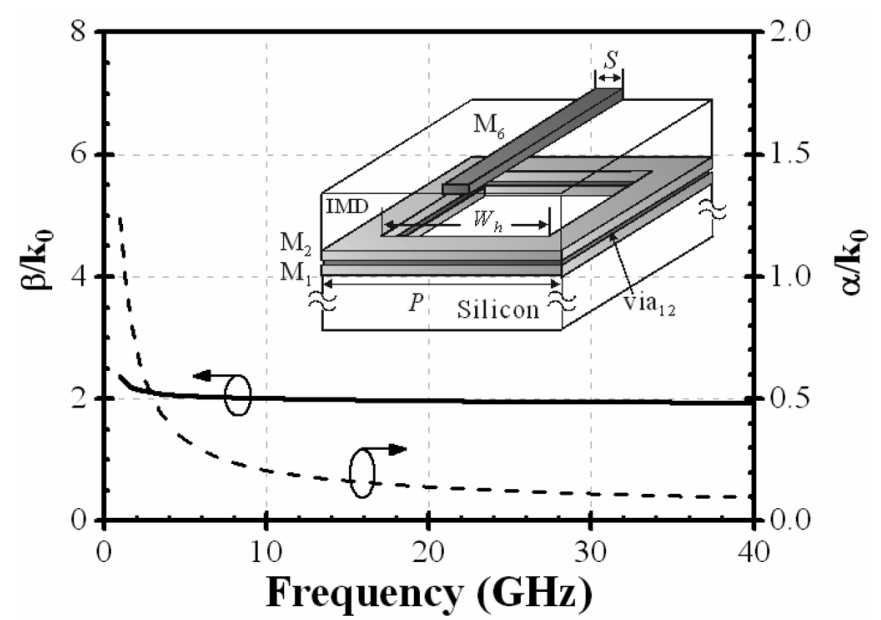

Fig. 2. Simulated normalized propagation constants (phase constant $\beta / k_{0}$ ) and attenuation constant $\left(\alpha / k_{0}\right)$ of the $71.8 \Omega$ quarter-wavelength CCS TL versus frequency. Structural parameters are $S=4.0 \mu \mathrm{m}, P=30.0 \mu \mathrm{m}$, and $W_{h}=$ $28.0 \mu \mathrm{m}$.

silicon substrate are $482.6 \mu \mathrm{m}$ and $11.0 \mathrm{~S} / \mathrm{m}$, respectively. The normalized complex propagation constant is extracted from the two-port EM simulations. Fig. 2 shows the normalized phase constant $\left(\beta / k_{0}\right)$ and normalized attenuation constant $\left(\alpha / k_{0}\right)$, revealing the following observations. First, the normalized phase constant of the CCS TL is about 1.94 at Ka-band, which approaches the physical limit of the TL in quasi-TEM mode. The loss of the CCS TL, which is inversely proportional to the square root of frequency, follows the physical phenomenon of skin effect. Consequently, the quality-factor ( $Q$-factor) of the CCS TL, which is defined by $Q=\beta / 2 \alpha$, is about 10.0 at Ka-band. The uniform characteristics of the CCS TL are applied to designing a two-way $3 \mathrm{~dB}$ Wilkinson power divider, which is reported in the next section.

\section{LOW LosS TWO-WAY 3 DB WILKINSON POWER DIVIDER DESIGN BY USING CCS TLS}

The design of two-way $3 \mathrm{~dB}$ power divider is followed by the well-documented text book [2, pp.318-322]. In the design, all three ports are designed to match the $50.0 \Omega$ system. Therefore, the characteristic impedance and resistance of two quarterwavelength TLs and the isolation resistor $\left(R_{I}\right)$ are $70.7 \Omega$ and $100.0 \Omega$, respectively, for achieving equal power-split. Furthermore, the effects of junctions, including of the feeding ports of the divider and the connections between the isolation resistor and the CCS TLs, are also considered. The parasitic eliminations lead the following fine adjustments for the presented design. The length of the CCS TL is reduced from $1127.0 \mu \mathrm{m}$ to $1080.0 \mu \mathrm{m}$, and the characteristic impedance of the CCS TL is increased from 70.7 to $71.8 \Omega$. The traces between the contact pads and divider are realized by $71.8 \Omega$ CCS TL with a length of $15.0 \mu \mathrm{m}$. The equivalent circuit of the proposed divider is constructed based on the parameters of the CCS TL reported in Fig. 2 and analyzed using commercial software, Agilent ADS $2006 \mathrm{~A}$, for making a quick assessment of the divider performances. Moreover, the prototype is fabricated by a standard $0.18 \mu \mathrm{m}$ 1P6M CMOS technology. As shown in Fig. 1, the chip area is $210.0 \mu \mathrm{m} \times 390.0 \mu \mathrm{m}$ without contact pads. The

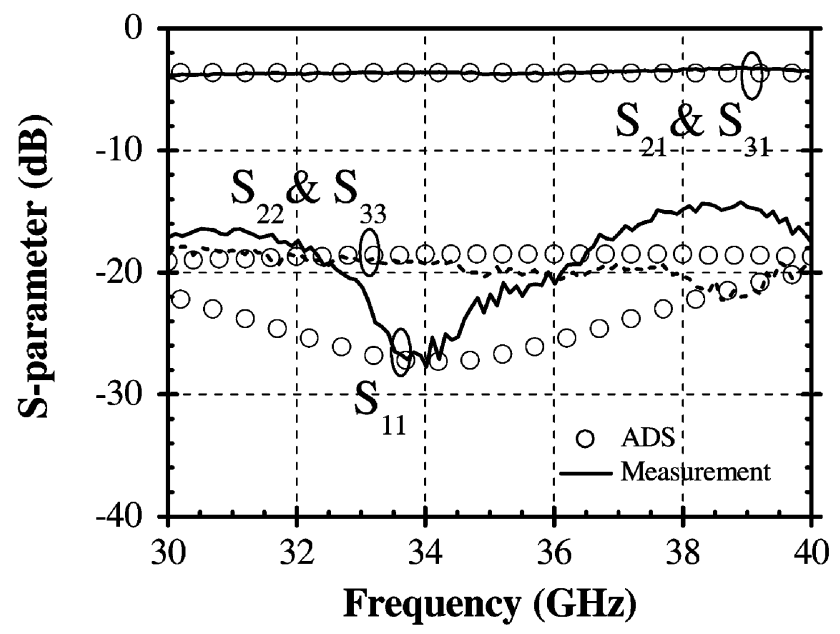

(a)

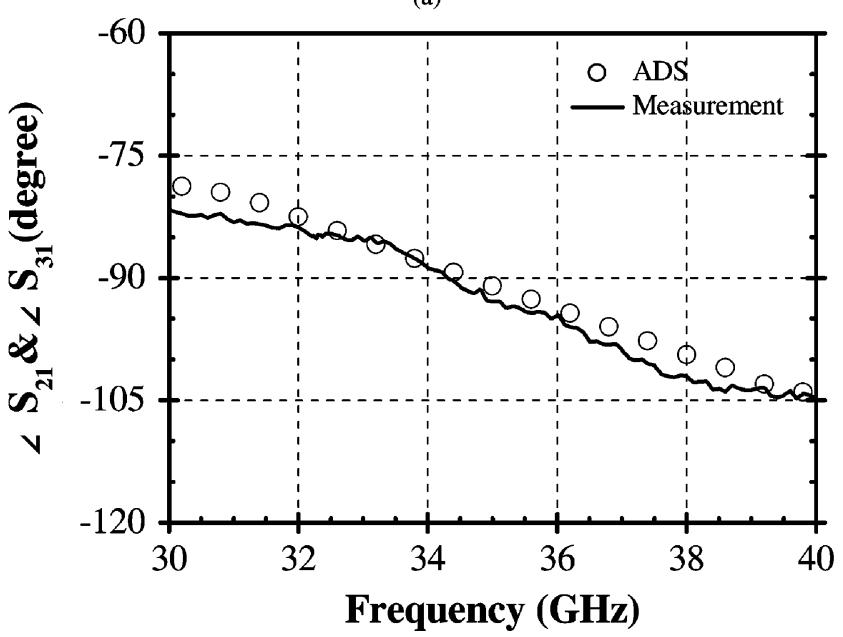

(b)

Fig. 3. Comparison between simulated and measured $S$-parameters of the prototype in Fig. 1: (a) magnitude and (b) phase.

layout of the prototype is kept symmetrical. The isolation resistor is realized by the POLY resistor. The width and length of the POLY resistor are $1.29 \mu \mathrm{m}$ and $2.18 \mu \mathrm{m}$, respectively. The on-chip measurements are performed by vector network analyzer (VNA) after the -open-load-through (SOLT) calibration procedures that had been performed.

Fig. 3(a) and (b) show the comparisons between the measured results and the circuit simulations. Since the prototype, as shown in Fig. 1, is symmetrical, the scattering parameters at port 2 and port 3 are identical. At $34.3 \mathrm{GHz}$, the measured insertion losses $\left(S_{21}\right.$ and $\left.S_{31}\right)$ of the power divider are $3.62 \mathrm{~dB}$, revealing only $0.62 \mathrm{~dB}$ higher than that of the loss-free performance. The measured insertion losses of the proposed power divider from 32.6 to $40.0 \mathrm{GHz}$ are less than $3.65 \mathrm{~dB}$ and the equal power split can be observed at this bandwidth. The circuit simulations indicate that the insertion losses are $3.64 \mathrm{~dB}$, showing excellent agreements with that of the measured results. The return loss of port 1 from 32.6 to $36.0 \mathrm{GHz}$ is higher than $20.0 \mathrm{~dB}$, showing a $9.9 \%$ bandwidth of the $34.3 \mathrm{GHz}$. The return losses of port 2 and port 3 of the prototype are higher than $15.0 \mathrm{~dB}$ from 30.0 to 40.0 GHz. On the other hand, Fig. 3(b) shows the phase of $S_{21}$ and $S_{31}$. Both simulation and measurement indicate a $90.0^{\circ} \mathrm{lag}$ 


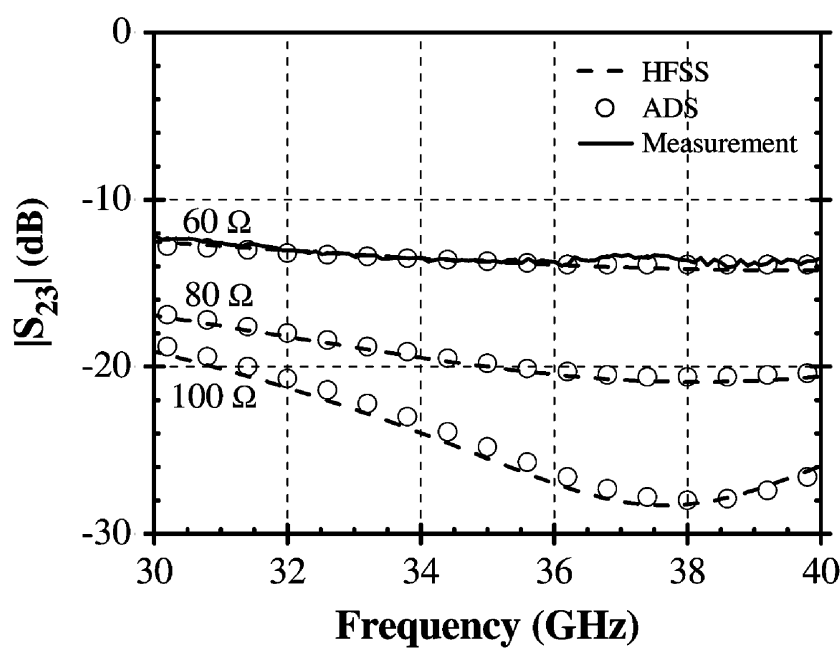

Fig. 4. Isolation between port 2 and port 3 versus the isolation resistances.

at $34.3 \mathrm{GHz}$. The comparisons between simulations and measurements shown in Fig. 3(a) and (b) confirm the guiding characteristics of the CCS TL shown in Fig. 2.

Fig. 4 shows the isolation between port 2 and port 3 . The measured result, which is the solid line in Fig. 4, shows a maximum isolation of $13.5 \mathrm{~dB}$ from 30.0 to $40.0 \mathrm{GHz}$, revealing a $15.0 \mathrm{~dB}$ difference from the ADS simulation with an isolation resistor $\left(R_{I}\right)$ of $100.0 \Omega$. However, the hollow circles, which represent the ADS simulation with $R_{I}$ of $60.0 \Omega$, show perfect agreements with those of the measured results. Such comparison implies that the value of the POLY resistor may be changed due to its parasitic effects, consistent with the extrapolated foundry data of $40.0 \%$ reduction in resistive value of a POLY resistor. Moreover, the completed prototype is analyzed by using full-wave EM simulator Ansoft HFSS to investigate the layout effects on the divider isolation. As shown in Fig. 4, the results of these two types of simulations, which are set up based on the identical structural and material parameters except the values of $R_{I}$, reveal excellent agreements with those of the measurements and circuit simulations. Such investigations confirm the resistances drift of $R_{I}$ in the prototype shown in Fig. 1.

\section{CONCLUSION}

A Ka-band two-way Wilkinson power divider, which is realized by the CCS TL, has been designed and fabricated by a standard $0.18 \mu \mathrm{m}$ 1P6M CMOS technology. The on-chip CCS TL is theoretically investigated by full-wave EM simulations.
The guiding properties of CCS TL, including complex propagation constant, characteristic impedance and quality factor, are extracted from the EM results and applied to the transmission model for the power divider design. The comparisons between the equivalent circuit simulations and on-chip measurements, which show perfect agreements, reveal the following characteristics of the proposed TL-based power divider. The divider is equally power-split and the intrinsic losses from 32.6 to $40.0 \mathrm{GHz}$ are less than $0.65 \mathrm{~dB}$. The return losses from 30.0 to $40.0 \mathrm{GHz}$ are higher than $10.0 \mathrm{~dB}$. Additionally, the measured results show that the isolation between two output ports is $13.5 \mathrm{~dB}$, which is $15.0 \mathrm{~dB}$ lower than that of the ADS simulation. A series of simulations confirm that such difference is caused by the resistance drift of the isolation resistor, which is realized by the on-chip poly lines. On the other hand, the size of prototype, which is realized only by CCS TL and requiring resistor, is $210.0 \mu \mathrm{m} \times 390.0 \mu \mathrm{m}$ without contact pads. Such device has high potential on the application of CMOS RF system-on-chip (SOC).

\section{REFERENCES}

[1] J. Wilkinson, "An n-way hybrid power divider," IEEE Trans. Microw. Theory Tech., vol. MTT-8, no. 1, pp. 116-118, Jan. 1960.

[2] D. M. Pozar, Microwave Engineering, 3rd ed. New York: Wiley, 2005 , ch. 7

[3] M. Ozgur, M. E. Zaghloul, and M. Gaitan, "Micromachined 28-GHz power divider in CMOS technology," IEEE Microw. Guided Wave Lett., vol. 10, no. 3, pp. 99-101, Mar. 2000.

[4] L.-H. Lu, P. Bhattacharya, L. P. B. Katehi, and G. E. Ponchak, "X-band and K-band lumped wilkinson power dividers with a micromachined technology," in IEEE MTT-S Int. Dig., 2000, pp. 287-290.

[5] T. Ji, H. Yoon, J. K. Abraham, and V. K. Varadan, "Ku-band antenna array feed with ferroelectric phase shifters on silicon distribution network," IEEE Trans. Microw. Theory Tech., vol. 54, no. 3, pp. 1131-1138, Mar. 2006

[6] M. C. Scardelletti, G. E. Ponchak, and T. M. Weller, "Miniaturized Wilkinson power dividers utilizing capacitive loading," IEEE Microw. Wireless Compon. Lett., vol. 12, no. 1, pp. 6-8, Jan. 2002.

[7] K. Hettak, G. A. Morin, and M. G. Stubbs, "Size reduction of a MMIC direct up-converter at $44 \mathrm{GHz}$ in multilayer CPW technology using thin-film microstrip stubs loading," IEEE Trans. Microw. Theory Tech., vol. 54, no. 9, pp. 3453-3461, Sep. 2006.

[8] K. Hettak, G. A. Morin, and M. G. Stubbs, "Compact MMIC CPW and asymmetric CPS branch-line couplers and Wilkinson dividers using shunt and series stub loading," IEEE Trans. Microw. Theory Tech., vol. 53, no. 5, pp. 1624-1635, May 2005.

[9] K. Nishikawa, T. Tokumitsu, and I. Toyoda, "Miniaturized Wilkinson power divider using three-dimensional MMIC technology," IEEE Microw. Guided Wave Lett., vol. 6, no. 10, pp. 372-374, Oct. 1996

[10] C.-C. Chen and C.-K. C. Tzuang, "Synthetic quasi-TEM meandered transmission lines for compacted microwave integrated circuits," IEEE Trans. Microw. Theory Tech., vol. 52, no. 6, pp. 1637-1647, Jun. 2004.

[11] S. Wang and C.-K. C. Tzuang, "Compacted Ka-band CMOS rat-race hybrid using synthesized transmission lines," in Proc. IEEE MTT-S Int. Dig., Honolulu, HI, 2007, pp. 1023-1026. 